Chirurg 2019 $\cdot 90: 416$

https://doi.org/10.1007/s00104-019-0933-1

Online publiziert: 14. März 2019

(c) Springer Medizin Verlag GmbH, ein Teil von Springer Nature 2019

\section{Originalpublikation}

Odensten C, Strigård K, Rutegård J et al (2019) Use of prophylactic mesh when creating a colostomy does not prevent parastomal hernia. Ann Surg 269:427-431. https://doi. org/10.1097/SLA.0000000000002542

Hintergrund. Die European Hernia Society empfiehlt in ihrer Leitlinie seit 2017 den prophylaktischen Einsatz nichtresorbierbarer Netze bei der elektiven Anlage eines endständigen Kolostomas (Starke Empfehlung, Evidenzgrad 4/4; [1]). Die Ergebnisse der hier vorgestellten, bisher größten multizentrischen und randomisierten Studie (STOMAMESH) widersprechen jedoch dieser Empfehlung.

Methoden. Es handelt sich um eine randomisierte multizentrische Studie, die von 2007 bis 2015 an acht schwedischen Zentren durchgeführt wurde. Patienten und Untersucher waren verblindet. Patienten mit vorausgegangener Stomaanlage, Peritonitis oder einem erwarteten Überleben von weniger als drei Jahren wurden ausgeschlossen. Die Kolostomaanlage ohne Netz erfolgte an präoperativ markierten Stellen durch den M. rectus abdominis. Die Stomaanlage mit Netz erfolgte unter Verwendung eines leichtgewichtigen Polypropylennetzes in der Sublay-Position.

Ergebnisse. Insgesamt wurden $232 \mathrm{~Pa}$ tienten randomisiert. Nach einem Jahr wurden 211 (91\%) der Patienten kli-

M. Schrempf $\cdot$ M. Anthuber

Klinik für Allgemein-, Viszeral-, Transplantationschirurgie, Universitätsklinikum Augsburg, Augsburg, Deutschland

\title{
Der prophylaktische Einsatz von Netzen verhindert nicht die Entstehung parastomaler Hernien beim endständigen Kolostoma
}

nisch und 198 (85\%) der Patienten mittels Computertomographie (CT) untersucht. Zwischen den Gruppen mit Netz und ohne Netz ergab sich kein Unterschied bezüglich der Rate an klinisch $(29 \%$ vs. $30 \%, p=0,866)$ und radiologisch $(32 \%$ vs. $34 \% p=0,765)$ nachgewiesenen Hernien. Die Rate an Komplikationen nach einem Monat unterschied sich zwischen den beiden Gruppen nicht $(p=0,668)$. Die Operationszeit in der Netz-Gruppe war durchschnittlich 36 min länger $(p=0,019)$.

\section{Diskussion und Fazit}

Die Ergebnisse dieser Arbeit stehen im Widerspruch zu den Empfehlungen der European Hernia Society und einer kürzlich publizierten Metaanalyse [3]. Stärken dieser Arbeit sind die Verblindung und das multizentrische Design, da viele der bisherigen Publikationen an spezialisierten Einzelzentren durchgeführt wurden. In der zweitgrößten Studie (PREVENT) von Brandsma et al., die einen Vorteil für die prophylaktische Netzimplantation zeigen konnte, erfolgte keine Verblindung der Untersucher und kein routinemäßiger Einsatz der CT-Diagnostik [2]. Diese zwei Faktoren könnten die Unterschiede zwischen den beiden Studien erklären. In der Diskussion um die prophylaktische Verwendung von Netzen kommt die Tatsache zu kurz, dass parastomale Hernien teilweise asymptomatisch sind und beispielsweise aufgrund von Tumorprogress in manchen Fällen keine therapeutische Relevanz haben. Zudem fehlen gute Langzeitdaten zu netzbedingten Komplikationen wie Darmwandarrosionen, Fistelbildung und Schmerzen. Diese müssten bei einem flächendeckenden prophylaktischen Einsatz von Netzen stärker berücksichtigt werden. Auf der Grundlage der Ergebnisse der STOMAMESH-Studie muss deshalb die aktuelle Empfehlung der European Hernia Society kritisch hinterfragt werden.

\section{Korrespondenzadresse}

\section{Prof. Dr. M. Anthuber}

Klinik für Allgemein-, Viszeral-, Transplantationschirurgie, Universitätsklinikum Augsburg

Stenglinstr. 2, 86156 Augsburg, Deutschland matthias.anthuber@klinikum-augsburg.de

Interessenkonflikt. M. Schrempf und M. Anthuber geben an, dass kein Interessenkonflikt besteht.

\section{Literatur}

1. Antoniou SA, Agresta F, Garcia Alamino JM et al (2018) European Hernia Society guidelines on prevention and treatment of parastomal hernias. Hernia 22:183-198. https://doi.org/10. 1007/s10029-017-1697-5

2. Brandsma HT, Hansson BME, Aufenacker TJ et al (2016) Prophylactic mesh placement to prevent parastomal hernia, early results of a prospective multicentre randomized trial. Hernia 20:535-541. https://doi.org/10.1007/s10029-015-1427-9

3. Cross AJ, Buchwald PL, Frizelle FA, Eglinton TW (2017) Meta-analysis of prophylactic mesh to prevent parastomal hernia. Br J Surg 104:179-186. https://doi.org/10.1002/bjs.10402 